\title{
Simulation and testing of a power converter for aircraft AC electric power generation system using software elimination of higher harmonics
}

Kulmanov, V., Anuchin, A., Ostrirov, V., Rusakov, A. and Vagapov, Y.

This is a paper presented at the 53rd IEEE Int. Universities Power Engineering Conference UPEC-2018, Glasgow, UK, 4-7 September 2018.

Copyright of the author(s). Reproduced here with their permission and the permission of the conference organisers.

Recommended citation:

Kulmanov, V., Anuchin, A., Ostrirov, V., Rusakov, A. and Vagapov, Y. (2018), Simulation and testing of a power converter for aircraft $A C$ electric power generation system using software elimination of higher harmonics. In: Proc. 53rd IEEE Int. Universities Power Engineering Conference UPEC-2018, Glasgow, UK, 4-7 September 2018, pp. 1-6. doi:

10.1109/UPEC.2018.8541922 


\section{Simulation and Testing of a Power Converter for Aircraft AC Electric Power Generation System using Software Elimination of Higher Harmonics}

\author{
Vasiliy Kulmanov \\ Department of Electric Drives \\ Moscow Power Engineering Institute \\ Moscow, Russia \\ kulmanovvi@mpei.ru
}

Anatoliy Rusakov

Department of Electrical Complexes of Self-

Contained Objects and Electrical Transport

Moscow Power Engineering Institute

Moscow, Russia

rusakovam@list.ru

\author{
Alecksey Anuchin \\ Department of Electric Drives \\ Moscow Power Engineering Institute \\ Moscow, Russia \\ anuchin.alecksey@gmail.com
}

\author{
Yuriy Vagapov \\ Department of Engineering \\ Glyndwr University \\ Wrexham, United Kingdom \\ y.vagapov@glyndwr.ac.uk
}

\author{
Vadim Ostrirov \\ Department of Electric Drives \\ Moscow Power Engineering Institute \\ Moscow, Russia \\ ostrirovvn@cycle-p.ru
}

\begin{abstract}
This paper presents the results of simulation and practical tests of the inverter-based power converter with a sine output filter designed for implementation in an aircraft power system. In order to reduce the output voltage harmonic distortion, the converter utilises active software harmonics filtering. This work provides an analysis of the converter performance under two control algorithms applied for the active filtering operation. The first control algorithm is based on discrete Fourier transform (DFT) where the control system injects measured higher harmonics with inverse polarity into the inverter command voltage and DFT provides the measurement of the converter output voltage. The second control method is based on repetitive controller (RC) algorithms which adjusts the inverter command voltage using a self-learning approach. The second method significantly reduces the demand for CPU computational resources. The software filtering in both systems shows a good performance and ensures the converter output voltage with less than $5 \%$ THD under all load conditions. This paper discusses the structures for both proposed control systems as well as the implementation in MATLAB models and microcontroller software. Both algorithms are modelled in software and implemented in a practical microcontroller. The advantages of the RC-based control method are demonstrated through the software analysis and performance of the system.
\end{abstract}

Keywords - aircraft power system, digital Fourier transform, higher harmonics filtering, power converters, power filters, repetitive control, total harmonic distortion

\section{INTRODUCTION}

The structure of conventional electrical power system in aircraft is a chain of electro-mechanical devices comprising of jet engine, constant speed drive and synchronous generator. The jet engine plays role of a prime mover and rotates its shaft at variable speed following the propulsion modes. The purpose of the constant speed drive is to convert mechanically the variable speed input into a constant speed output to provide rotation of the synchronous generator shaft at a fixed velocity. The constant speed drive is most complicate, expensive and

The research was performed with the support of the Russian Ministry of Education and Science grant (Project № 8.8313.2017/BCh). unreliable link in the electro-mechanical chain of power conversion. It costs about $80 \%$ of total generating equipment price and takes about $80 \%$ of its total service time [1].

Due to advances in the area of power electronic it is suggested to remove the constant speed drive from the chain, connect the synchronous generator directly to the jet engine shaft and implement an additional power electronic component, frequency converter. Under this scheme, the variable frequency $\mathrm{AC}$ voltage produced by the synchronous generator is converted by the power electronic circuit into AC electrical output at constant frequency required for the normal operation of the aircraft. This approach significantly improves the reliability and efficiency of the conversion of mechanical power into electricity. The proposed scheme is shown in Fig. $1 b$.

Quality of power produced by the converter must be in accordance to the existing standards [2]:

- Connection: 3 line wires +1 neutral wire.

- THD: $5 \%$ or less.

- Output voltage magnitude: 1.31 to 1.51 of rated voltage.

- Operation under unbalanced and rectifier loads.

Power converter must have maximum power-to-weight ratio. The suggested topology of the frequency converter shown in Fig. 2 provides the three-phase output with neutral to energize electrical loads of aircraft equipment.
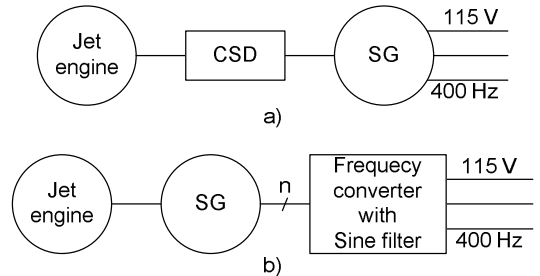

Fig. 1. Aircraft AC generation systems. (a) conventional and (b) modern 


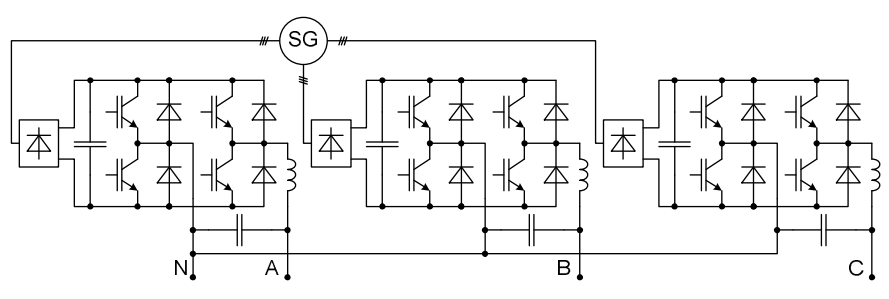

Fig. 2. Topology of the frequency converter for aircraft power system

The main sources of harmonic distortion in a frequency converter are the influences of dead time and non-linear voltage drop across a sine filter (under non-linear load conditions). Conventional techniques for dead time compensation [3], [4] do not work stable in this converter because of strong current pulsation across the zero value caused by small sine filter inductance.

Existing control strategies for voltage-source inverters described in [5], [6], [7] have some drawbacks. The absence of a zero wire in all these inverters contradicts the requirements of the standard. Space vector PWM used in [5] is incompatible with proposed frequency converter topology. The sine filter reactors with high inductance discussed in [5], [7] (also probably in [6] although it is not mentioned), leads to an increase in the converter weight. Solutions suggested in [5], [7] imply the use of a powerful processors with $1 \mathrm{GHz}$ clocking frequency, therefore the algorithms demand the significant CPU computational resources. If the Motor Control series microcontroller (which has all necessary peripheral devices and is relatively cheap) is used, these algorithms probably may not be realised (especially with five time higher carrier frequency than in [5]). In [6] a derivative voltage feedback is used. It can cause system instability due to noise in analog signal. A control system with higher harmonics correction based on digital Fourier transform was designed to solve these problems.

Each phase of the frequency converter is controlled and operates independently. Hence, it is rational to consider and simulate only one phase of the converter. The single-phase topology is presented in Fig. 3.

The variable speed multi-phase generator driven by jet engine is connected to the input. Each phase of the converter consists of a three-phase uncontrolled bridge rectifier which charges a DC link filter capacitor and a single-phase full-bridge IGBT inverter which converts DC voltage into $115 \mathrm{~V}, 400 \mathrm{~Hz}$ sinusoidal output voltage with PWM ripples filtered by a sine output filter. In addition to PWM ripples the output voltage is distorted by some higher harmonics caused by inverter nonlinearity such as dead time and non-linear load (rectifiers). To meet the voltage quality requirements of the standard inverter output voltage should be filtered via a combination of power and software tools. A sine filter with cutoff frequency of about $1 / 4$ of carrier frequency is used to suppress PWM switching ripple. A software algorithm adjusting inverter commanded value curve according to voltage measured on previous period of fundamental frequency in order to eliminate low-order higher harmonics complements the sine filter.

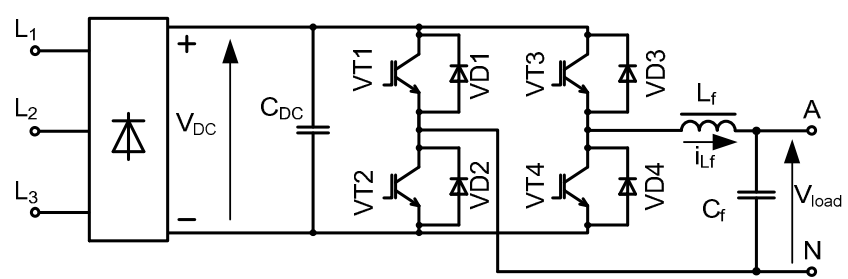

Fig. 3. Frequency converter schematics (single-phase)

\section{FREQUENCY CONVERTER MODEL}

Simulation was performed in Simulink environment of MATLAB. The converter model consists of two main parts: a power unit and a control system block. This section discusses the power circuitry model consisting of a $190 \mathrm{~V}, 1500 \mathrm{~Hz} \mathrm{AC}$ generator, and the frequency converter. There are some current and voltage sensors for measuring operating values for control system feedbacks and graphic scopes.

Inverter PWM frequency is $25.6 \mathrm{kHz}$. This frequency is chosen specifically to be an integer multiple of the fundamental frequency of the aircraft power system - it is 64 times higher than $400 \mathrm{~Hz}$. This is an important approach for higher harmonics correction algorithms.

The model takes into account the ripples of the rectified voltage presented in the practical frequency converters. Measured DC link voltage is used by a control system as a feedback for this ripple compensation algorithm: when the duty cycle is calculated, it is divided by the value of the measured voltage $\mathrm{V}_{\mathrm{DC}}$. A microcontroller $\mathrm{ADC}$ operation is simulated by a zero order hold unit. Data received from the current and voltage sensors are supplied to the microcontroller in the form of samples at constant intervals. The sampling period is adjusted in the $\mathrm{ZOH}$ unit. The value of the inverter command voltage is calculated by the control system software in the Sfunction block but the duty cycle is calculated using the math elements of the Simulink library for simplicity. A PWM generator converts numeric duty cycle values into PWM signals taking into account the adjusted dead time. The PWM signals are fed to the control inputs of inverter IGBTs as shown in Fig. 4.

The dead time of $2.5 \mu \mathrm{s}$ is enough for the modern IGBTs. But it is still a strong disturbing factor for the output voltage waveform because of high carrier frequency. An open-loop control system without any harmonic correction was simulated at the first stage to estimate a value of harmonic distortion presented in the output voltage (see graphs in Fig. 6).

As can be seen in these plots the output voltage is distorted because of the difference between the calculated and the real duty cycle which is caused mainly by the inverter dead time. This effect is most noticeable in areas where the current crosses the zero value (see areas A and B in Fig. 6). Such a strong influence of the dead time is explained by a high ratio of dead time value and the PWM cycle value. The dead time value of $2.5 \mu$ s equals to $6.4 \%$ of the PWM period at the chosen frequency of $25.6 \mathrm{kHz}$. A high carrier frequency is required for the proper modulation of $400 \mathrm{~Hz}$ load voltage and for the output filter weight reduction as well. 


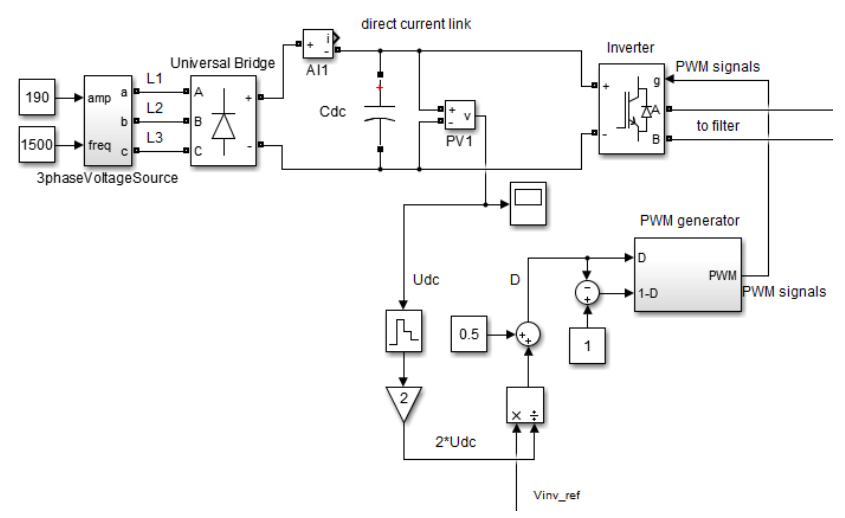

Fig. 4. Generator and frequency converter model

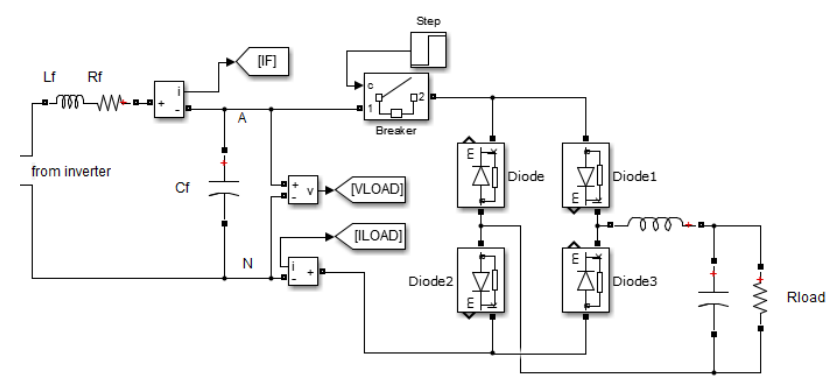

Fig. 5. Sine output filter and rectifire load model
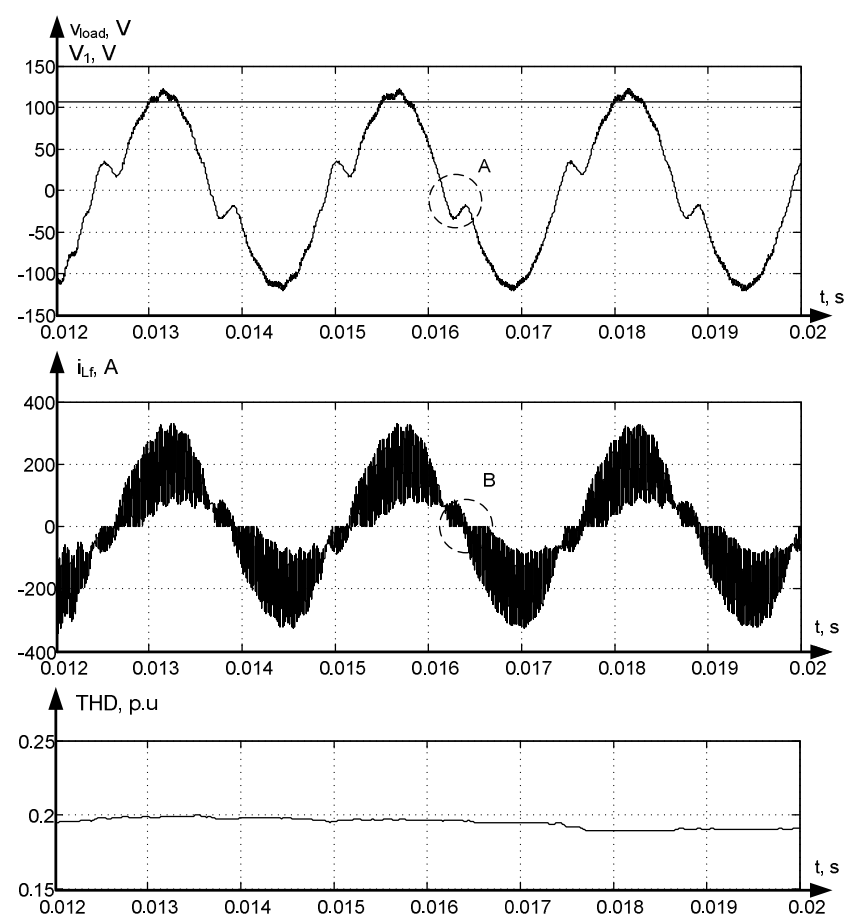

Fig. 6. Output voltage and it's fundamental magnitude (top), filter reactor current (middle) and THD (bottom) for open-loop control system

As can be seen in the bottom plot of Fig. 6 the THD reaches almost $20 \%$, which is absolutely unacceptable for aircraft electrical system. The Russian standard for aircraft electric supply systems requires THD of no more than 5\% for linear loads and $8 \%$ for non-linear ones.

The control system should be improved by software output voltage waveform correction algorithm. There are some odd higher harmonics (3rd, 5th, 7th etc.) in the load voltage caused by the dead time and non-linear loads. Therefore, the correction algorithm must be able to suppress these harmonics to achieve required electrical power quality.

\section{DFT-BASED CONTROL SYSTEM}

The inverter power supplies with sine output filters have the problems described in the previous paragraphs. In this paragraph an advanced version of the control system will be considered. It is able to suppress output voltage higher harmonics without using any additional power filters. The correction of higher harmonics is achieved by their estimation and injection into the inverter voltage commanded value curve with the opposite phase. So the load voltage has sinusoidal waveform.

Higher harmonics components can be estimated using DFT of the measured converter output voltage. Discrete Fourier transform algorithm can be performed in real time using modern motor control microcontrollers. Software DFT unit takes the measured voltage $v_{\text {load }}$ as input and calculates a Fourier series for it. So, at the output of DFT unit we have a sequence of harmonic component amplitudes. Block diagram of the DFT-based control system is shown in Fig. 7.

Discrete Fourier transform is represented by equations (1) and (2). So, they should be implemented in control system software.

$$
\begin{aligned}
& V_{n \cdot \cos }=\frac{2}{N} \sum_{i=1}^{N} v_{\text {load }}(i) \cdot \cos \left(n \cdot \frac{i}{N} \cdot 2 \pi\right) \\
& V_{n \cdot \sin }=\frac{2}{N} \sum_{i=1}^{N} v_{\text {load }}(i) \cdot \sin \left(n \cdot \frac{i}{N} \cdot 2 \pi\right)
\end{aligned}
$$

Sine and cosine component of each harmonic are calculated as finite sum of $N$ products of measured voltage and corresponding reference curve. For one whole period of fundamental harmonic $N$ samples of $v_{\text {load }}$ are taken. Samples multiplied by a required reference curve values are accumulated. Reference curves for compensated harmonics are stored in microcontroller memory as a table of sine and cosine function values. They are pre-calculated to optimize the control system requirements for processor resources. At the end of the fundamental period accumulated sums of $N$ products of $v_{\text {load }}$ samples and the table data points are numerically equal to corresponding harmonics magnitudes. The goal of the control system is to maintain fundamental harmonic at the nominal value and to reduce the most significant higher ones to zero (odd higher harmonics from the $3^{\text {rd }}$ to $9^{\text {th }}$ cause the strongest distortion in this case). So, DFT sums have to be calculated for fundamental harmonic sine component and for listed higher harmonics sine and cosine components. 


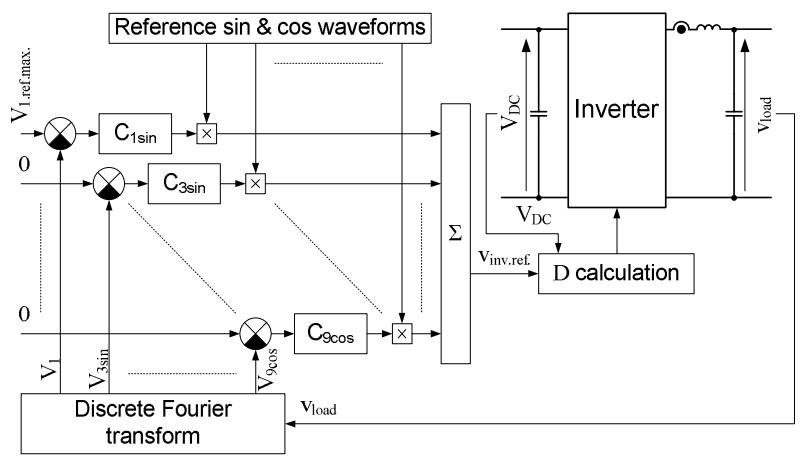

Fig. 7. DFT-based control system with higher harmonis compensation

The obtained at the output of DFT unit values of controlled harmonics magnitudes $\left(V_{l}, V_{3 \cos }, V_{3 \sin }, \ldots, V_{9 \cos }, V_{9 \sin }\right.$ in Fig. 7) are used for calculation of the required magnitudes which they must have in the voltage control signal. An array of conventional integral controllers is used to calculate these components. Calculated harmonics magnitudes are fed to the negative feedback inputs of integral controllers. Commanded value input of fundamental harmonic is a nominal value $V_{\text {Iref.max }}$, and all other harmonics controllers have a zero commanded value. Inverter voltage commanded value curve $V_{\text {inv.ref. }}$ is formed by a sum of reference harmonics multiplied by corresponding integral controller outputs (see Fig. 7). And then it is used to calculate a duty cycle for inverter power switches. In this way an output voltage waveform correction by an injection of higher harmonics in opposite phase is carried out.

\section{RC-BASED CONTROL SYSTEM}

Though the control method described above provides good quality of produced electric energy, it has one significant drawback - quite high requirements to microcontroller performance. As a result, it became possible to realize it only after deep optimization of program code including assembly language programming despite the use of one of the top microcontrollers from Texas Instruments.

After some review of papers on repetitive control ([8], [9], [10], and [11]) it was decided to apply such control strategy to the frequency converter. It is very promising in terms of reducing the requirements for the microcontroller.

The basic principle of the repetitive control system operation is as follows: output voltage at each point $(i=0 . .64)$ of next $(k+1)$ period of fundamental harmonic is corrected using the result of measurement at this point of current $(k)$ period. In other words, there is a personal integral controller for each time quantum inside the period. So, if we know that voltage at the point number $i$ in the previous fundamental period was less than the desirable value, we should increase the commanded value for the inverter at the point number $i$ in the current fundamental period and vice versa.

But there are some problems with the convergence of the control process in a real converter. The first problem occurs because of the transport delay which consists of ADC processing time and the inverter response to the applied control signal time. As a result, if the commanded value for the inverter is changed at the point number $i$, the output voltage will change somewhere at the point number $i+n$. In this frequency converter the delay depends mainly on the PWM period.

The phase-lead correction is used for transport delay compensation [8]. The idea of the correction is to take the commanded value for the inverter at the point number $i$ from the "future" output of the repetitive controller: at the point number $i+n$. For RC-based control system diagram see Fig. 8 .

The operating principle of the repetitive control system is briefly considered below. At the point number $i$ of the current period number $k$ the measured voltage is subtracted from a commanded value of the point number $i$. The difference is fed to the input of the repetitive controller. The inverter voltage commanded value in the next period at the point $i$ is taken from the output of the repetitive controller at the point $i+n$. An anticipation $n$ is set in PWM periods and equals to 2: $1 \mathrm{~T}_{\mathrm{PWM}}$ for $\mathrm{ADC}$ processing plus $1 \mathrm{~T}_{\mathrm{PWM}}$ for inverter response.

Another problem is the repetition process divergence in the high-frequency region. Simultaneously with the rapid convergence of the repetition process in the low-frequency region, a slower divergence occurs in the high-frequency region. To solve this problem a parallel correction with digital filter is used [8].

The conversion of the filter transfer function to a discrete domain and the difference equation are presented below.

$$
\begin{gathered}
Y(\mathrm{p})=X(\mathrm{p}) \frac{2}{K_{f}+2}-X(\mathrm{p}) \frac{e^{-\mathrm{p} T_{f}}}{K_{f}+2}-X(\mathrm{p}) \frac{e^{+\mathrm{p} T_{f}}}{K_{f}+2} \\
y[k]=\frac{2}{K_{f}+2} x[k]-\frac{1}{K_{f}+2}\left(x[k] \cdot \mathrm{z}^{-1}+x[k] \cdot \mathrm{z}^{+1}\right) \\
y[k]=\frac{2}{K_{f}+2} x[k]-\frac{1}{K_{f}+2}(x[k-1]+x[k+1]) .
\end{gathered}
$$

The control system model includes a sine block as a voltage reference, an output voltage sensor and a repetitive control system software calculating commanded value which is implemented in S-function unit.

The control system core is processed in the microcontroller interrupt at the end of PWM cycle. A maximum of $5859 \mathrm{CPU}$ clock cycles are available for interrupt handling with selected microcontroller clocking frequency of $150 \mathrm{MHz}$ and a PWM frequency of $25.6 \mathrm{kHz}$. RC-based control system core contains only a few fast operations and does not exceed a hundred of CPU cycles. This is much less than the DFT calculation or some microcontroller software service functions. Furthermore, the repetitive algorithm does no calculations in the ADC interrupt service routine while the DFT algorithm does. The $\mathrm{ADC}$ interrupt is 4 times more frequent than the PWM interrupt and the available for its processing time is four times less than in PWM interrupt (1464 cycles). 


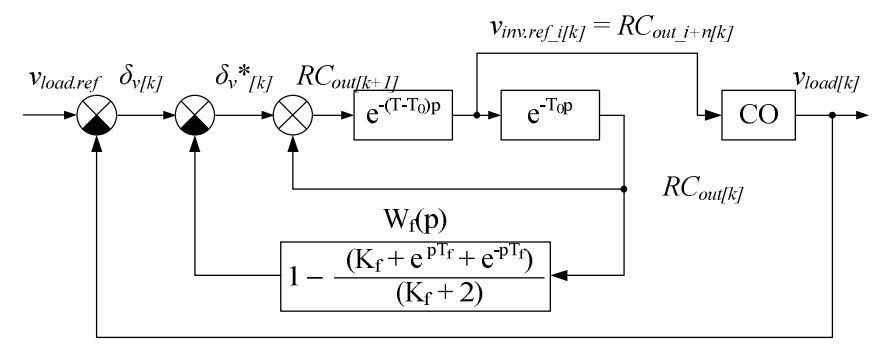

Fig. 8. RC-based control system with phase-lead and parallel correction

\section{PERFORMANCE TESTS}

The results of the MATLAB modelling and simulation of the converter operating using the proposed control algorithms show that the total harmonic distortion does not exceed $4 \%$ under all possible performance conditions. Both solutions are compared by two main parameters: the output voltage fundamental harmonic magnitude and THD. There are additional parameters for more convenient visualization on the graphs: the output voltage and the load current.

The performance of repetitive control system is not inferior to the DFT-based system which is illustrated by the following figures (Fig. 9 - Fig. 14). The RC-based system higher harmonics correction is slightly more accurate because it is not limited by a specific series of harmonics. So it suppresses distortion from all possible sources. All electric energy quality parameters meet the requirements of the standard [2].
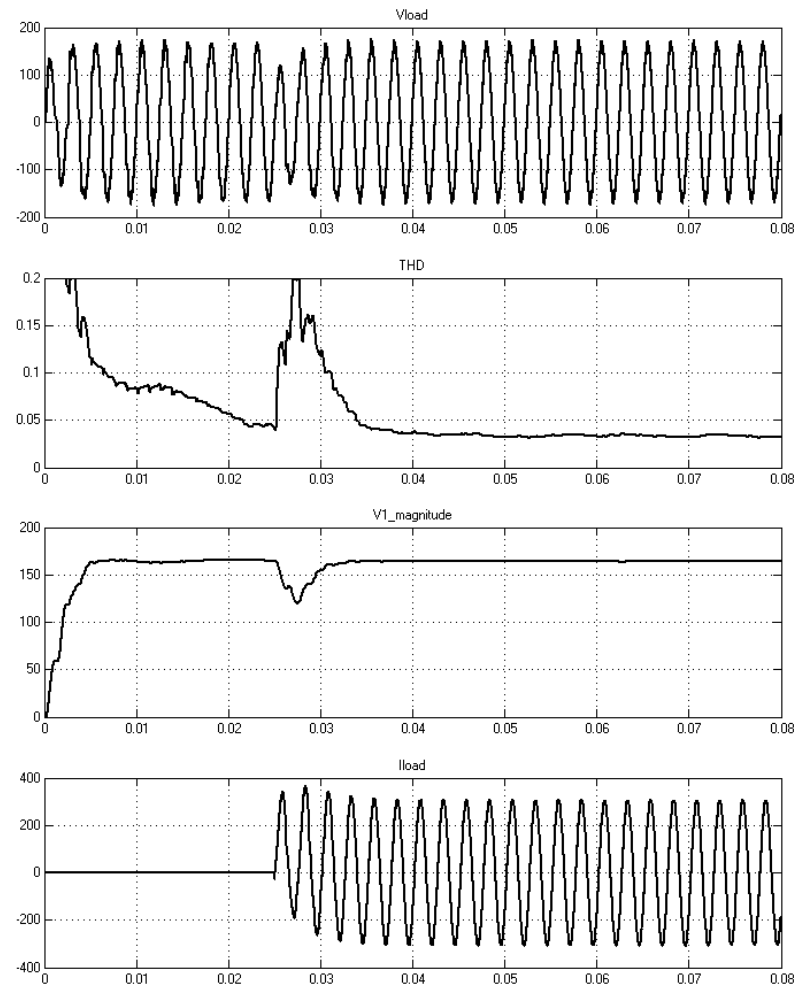

Time offset: 0

Fig. 9. DFT-based control system performance during RL-load step
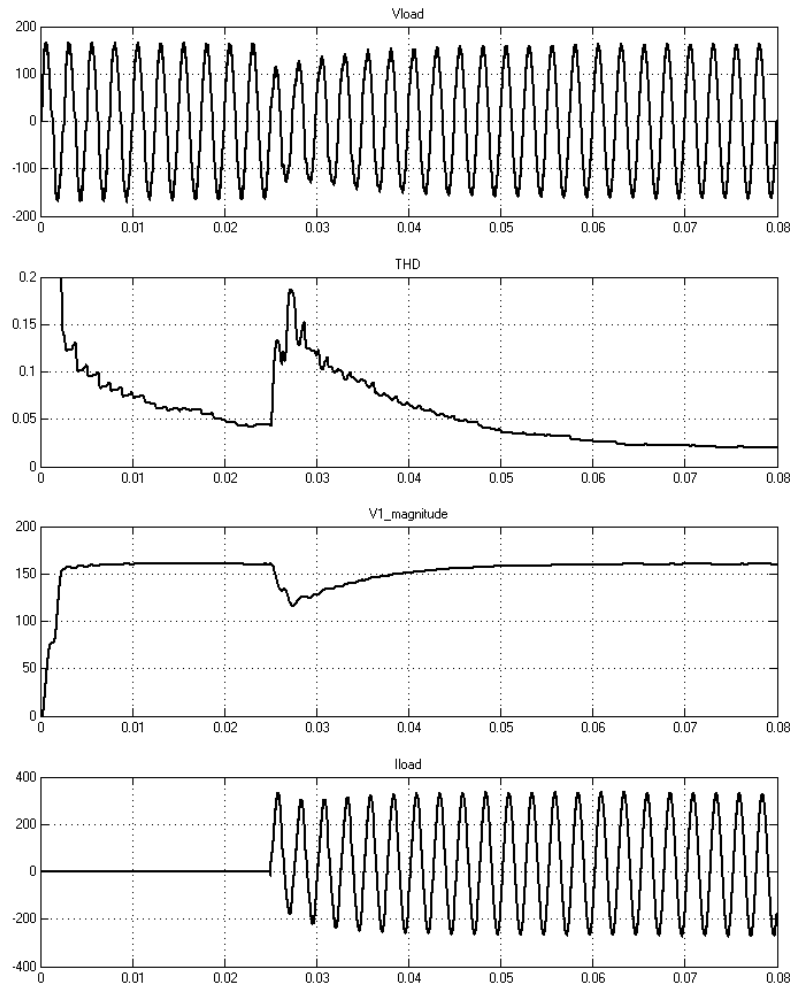

Time offset: 0

Fig. 10. RC-based control system performance during RL-load step
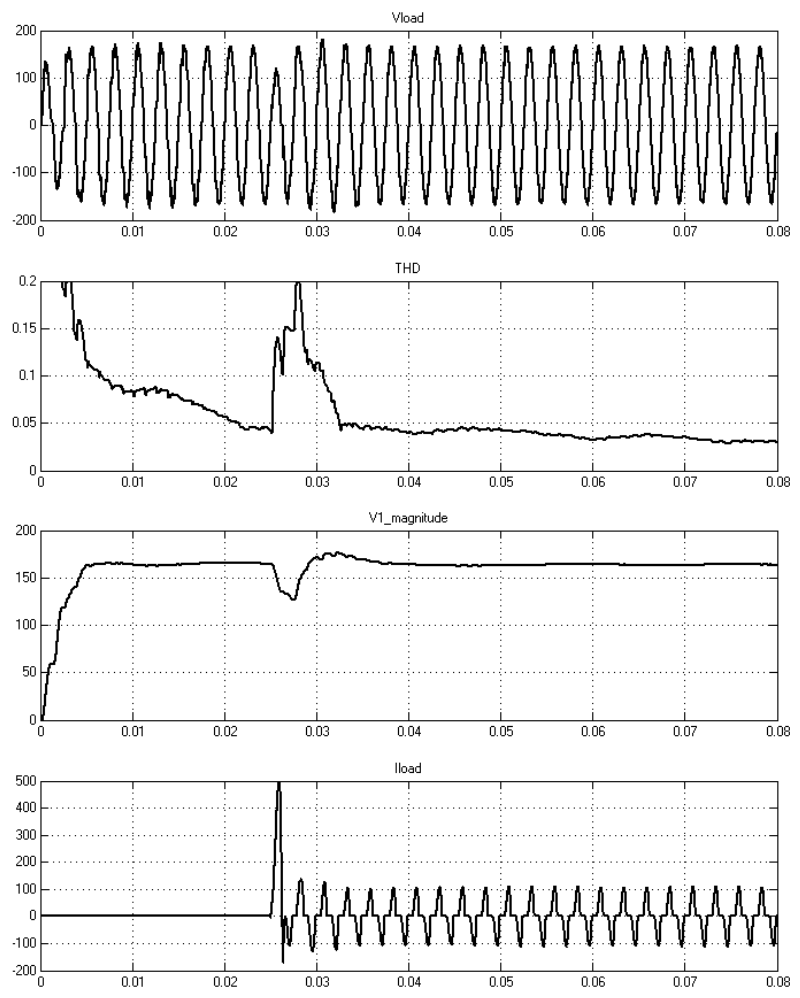

Time offset: 0

Fig. 11. DFT-based control system performance during rectifier load step ( $25 \%$ of rated power) 

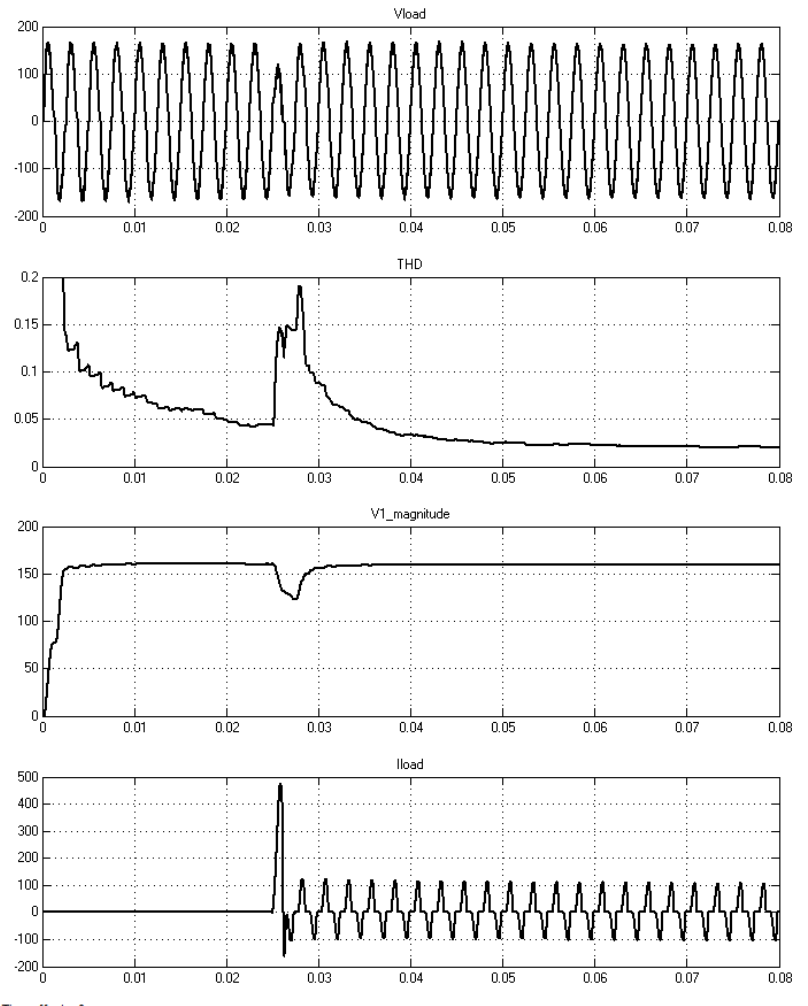

Time offset: 0

Fig. 12. RC-based control system performance during rectifier load step $(25 \%$ of rated power)
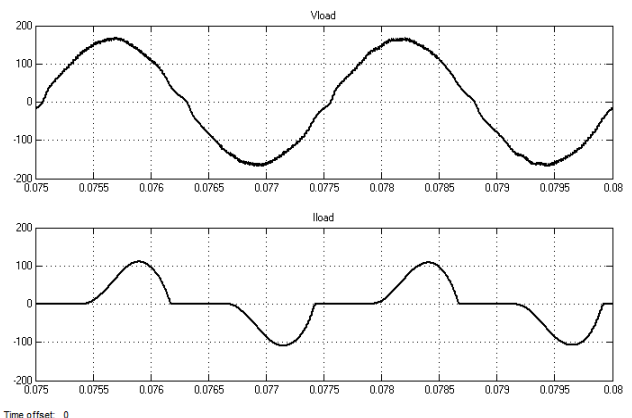

Fig. 13. Load voltage and current waveforms under rectifier load $(25 \%)$ in DFT-based control system
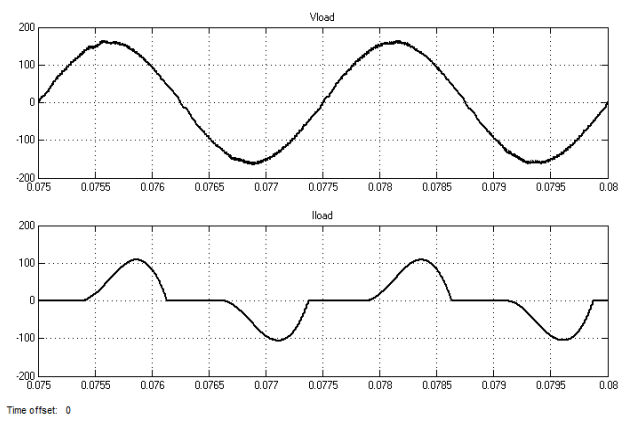

Fig. 14. Load voltage and current waveforms under rectifier load (25\%) in RC-based control system
The graphs of load voltage and current during the operation with rectifier load show that RC-based control system is able to eliminate some slight high-frequency distortions that are not suppressed by the DFT-based control system - see Fig. 13 and Fig. 14.

\section{CONCLUSIONS}

It is now possible to carry out full modernization of power supply system of aircraft to improve efficiency and increase reliability by means of electrical frequency conversion instead of the hydromechanical one used at present. The converter and control system that have been developed provide harmonic distortion of no more than $4 \%$ in all regimes of operation. The system quickly works through an increase and decrease in the load and, arriving at a steady state regime, takes up no more than five periods of the fundamental frequency.

\section{REFERENCES}

[1] I.M. Sindeev, and A.A. Savelov, Electric Supply Systems of Aircrafts. Moscow: Transport, 1990.

[2] Electric Supply Systems of Planes and Helicopters/Common Demands for Power Quality, GOSTR 54073-2010, 2011.

[3] L. Chen, and F.Z. Peng, "Dead-time elimination for voltage source inverters," IEEE Trans. Power Electronics, vol. 23, no. 2, pp.574-580, 2008.

[4] S.-G. Jeong, and M.-H. Park, "The analysis and compensation of deadtime effects in PWM inverters," IEEE Trans. Industrial Electronics, vol. 38, no. 2, April 1991.

[5] S. Piasecki, M. Jasinski, K. Rafal, M. Korzeniewski, and A. Milicua, "Higher harmonics compensation in grid-connected PWM converters for renewable energy interface and active filtering," Prezeglad Elektrotechniczny (Electrical Review), vol. 78, no. 6, pp. 85-90, 2011.

[6] A. Kulka, T. Undeland, S. Vazquez, and L.G. Franquelo, "Stationary frame voltage harmonic controller for standalone power generation," in Proc. European Conf. on Power Electronics and Applications, Aalborg, Denmark, 2-5 Sept. 2007, pp. 1-10.

[7] A. Micallef, M. Apap, C. Spiteri-Staines, J.M. Guerrero, J.C. Vasquez, "Reactive power sharing and voltage harmonic distortion compensation of droop controlled single phase islanded microgrids," IEEE Trans. Smart Grid, vol. 5, no. 3, pp. 1149-1158, 2014.

[8] A.A. Nikolskiy, Precise Self-learning Electric Drives for Non-circular Turning Lathes. Moscow: Advanced Solutions, 2016.

[9] S. Chen, Y.M. Lai, S.-C. Tan, and C.K. Tse, "Optimal design of repetitive controller for harmonic elimination in PWM voltage source inverters", in Proc. 29th Int. Telecommunications Energy Conf. INTELEC 2007, Rome, Italy, 30 Sept.-4 Oct. 2007, pp. 236-241.

[10] Y. Wan, F. Gao, and F.J. Doyle III, "Survey on iterative learning control, repetitive control, and run-to-run control," Journal of Process Control, vol. 19, no. 10, pp. 1589-1600, Dec. 2009.

[11] M. Steinbuch, "Repetitive control for systems with uncertain periodtime," Automatica, vol. 38, no. 12, pp. 2103-2109, Dec. 2002. 